\title{
Bound States at Threshold in Supersymmetric Quantum Mechanics
}

\author{
Massimo Porrati卬 \\ CPHT Ecole Polytechnique \\ F-91128 Palaiseau CEDEX, France \\ and \\ Department of Physics, NYU, 4 Washington Pl., New York, NY 10003, USA \\ and \\ Rockefeller University, New York, NY 10021-6399, USA \\ and \\ Alexander Rozenbergef \\ Department of Physics, NYU, 4 Washington Pl., New York, NY 10003, USA
}

\begin{abstract}
We propose a general method that allows to detect the existence of normalizable ground states in supersymmetric quantum mechanical systems with non-Fredholm spectrum. We apply our method to show the existence of bound states at threshold in two important cases: 1) the quantum mechanical system describing $\mathrm{H}$-monopoles; 2) the quantum mechanics of D0 branes.
\end{abstract}

\footnotetext{
${ }^{1}$ e-mail massimo.porrati@nyu.edu

${ }^{2}$ Permanent address.

${ }^{3}$ e-mail sasha.rozenberg@nyu.edu
} 


\section{Introduction}

In recent times, our knowledge of non-perturbative string theory has greatly progressed. A key element in this progress has been our ability to identify certain quantum mechanical (or fieldtheoretical) systems which describe non perturbative states in string theory. More precisely, BPS states in a given string model are often put in one-to-one correspondence with the ground states of some associated supersymmetric system. Two examples are paramount: the quantum mechanical reduction of a $6-\mathrm{d}, \mathrm{N}=1$ supersymmetric ( 8 real supercharges) field theory with one Abelian vector multiplet, and a charged hypermultiplet, and the quantum mechanical reduction of a $\mathrm{d}=10, \mathrm{~N}=1$ supersymmetric (16 real supercharges) $S U(N)$ gauge theory. The first system describes essentially an H-monopole [1] of the $\mathrm{SO}(32)$ superstring, while the second describes $N$ D0 branes. In both cases string duality arguments predict the existence of exactly one bound state at threshold for these systems, since string dualities map the BPS states they describe into Kaluza Klein modes of the graviton. To check the existence of these ground states at threshold directly in the QM model is thus a powerful check of the correctness of the duality hypothesis; the existence of a normalizable bound state for any $N$ in the D0 brane system is also essential for the consistency of the $\mathrm{M}$ (atrix) theory proposal of ref. [2].

The problem in counting the ground states arises from the fact that they are at threshold f This is because in the QM models described above, the bosonic coordinates take value in a non-compact manifold, and the potential energy has zero-energy valleys extending to infinity.

A possible way of counting the number of normalizable ground states (better, the difference between the multiplicity of bosonic and fermionic ground states), without explicitly solving the Schrödinger equations is to use the Witten index $W[\beta]=\operatorname{Tr}(-)^{F} \exp (-\beta H)$ [5], and to notice that when the low-energy spectrum is not too pathological, and $\operatorname{tr}(-)^{F} \exp (-\beta H)$ is trace-class, the following identity holds: $n_{B}-n_{F}=\lim _{\beta \rightarrow \infty} W[\beta]$. Here, $\operatorname{Tr}$ denotes the trace over the whole Hilbert space while tr is the trace over the (finite-dimensional) fermionic Hilbert space. Moreover, when the Hamiltonian of a supersymmetric QM has a continuous spectrum without a gap at zero energy, the usual arguments that ensure the invariance of the Witten index [5] have to be carefully re-examined. In refs. [6, 7], such analysis has been performed for the H-monopole, and for the D0-brane Hamiltonian with $N=2$ (see also $\mathbb{8}$ ). The result of refs. [6, [7, 8] is that in both cases $\lim _{\beta \rightarrow \infty} W[\beta]=1$.

In this paper, using a different method, we find that $n_{B}-n_{F}=1$ in the H-monopole system. We also find that the same result holds for D0 branes in 9 dimensions, provided that threedimensional D0 branes have no bound states?. Our method complements the arguments of

\footnotetext{
${ }^{4}$ For the D0 brane, this has been proven rigorously in [3, 4 .

${ }^{5}$ In ref. [9] it was proven rigorously that no bound states exist for D0 branes in two dimensions. An argument due to S. Shenker shows that a similar result should also hold in three dimensions, see also 10 .
} 
refs. [6, 7] since it proves the existence of D0 brane bound states with $N$ any prime number. Moreover, it appears to be straightforwardly generalizable to other, more complicated QM systems relevant to non-perturbative string theory. One chief example is the QM model of ref. [11, which is the reduction of a $4-\mathrm{d} N=1$ supersymmetric theory, and describes a configuration of four three-branes corresponding to a BPS black hole with nonzero entropy.

To completely check the prediction of duality, one should find a vanishing theorem proving the uniqueness of the bound states. Unfortunately, such a theorem does not exist yet in the literature. On the other hand, progress towards proving the absence of D0 brane bound states in three dimensions were recently made in [12].

The main idea of this paper is the following. In a supersymmetric QM with at least two (real) supercharges, there exists a real superpotential function $W$ [13]. A change in the superpotential, $w$, induces a change in the supercharges as follows:

$$
Q \rightarrow e^{-w} Q e^{w} \equiv Q_{w}, \quad \bar{Q} \rightarrow e^{w} \bar{Q} e^{-w} \equiv \bar{Q}_{w}
$$

If the bosonic fields take value in a compact space, or if $w / W$ goes to zero at infinity in field space, the $L_{2}$ cohomologies of $Q$ and $Q_{w}$ coincide, and therefore the number of normalizable ground states of the QM with superpotential $W+w$ is the same as for superpotential $W$ [5]. When the field space is non-compact, and the potential obtained from $W$ thas zero-energy valleys, the argument does not work, generically: the local cohomologies of $Q$ and $Q_{w}$ coincide, but the $L_{2}$ ones do not. Typically, one of the two cohomologies is normalizable while the other is not. In this paper, we show that, nevertheless, in the case of the H-monopole and D0 brane, it is possible to find an appropriate $w$ which acts as a "dam" for the valleys, lifting the flat directions in the potential, while maintaining the normalizability properties of the cohomology. This special perturbation allows us to reduce the computation of the index $n_{B}-n_{F}$ to computing the Witten index of a system with mass gap and isolated minima in the scalar potential. Such index can then be computed in the semiclassical approximation.

The paper is organized as follows. In Section 2, we describe more precisely the cohomology argument explained above. In Section 3, we find the cohomology-preserving perturbation $w$ for the H-monopole QM, while the perturbation for the D0 brane QM is discussed in Section 4, together with an argument showing that no bound states exist in $d<5$. Section 5 contains our conclusions and a discussion of possible generalizations. Some technical results are collected in the Appendices. Appendix A contains a discussion about the validity of the adiabatic (BornOppenheimer) approximation used in this paper, and an argument to justify it. Appendix B discusses some aspects of the semiclassical approximation in supersymmetric QM, which are used in Section 4. The eigenvalues of the fermion mass matrix of the perturbed D0 brane at

\footnotetext{
${ }^{6}$ Together with appropriate D-terms $[13$.
} 
the stationary point are computed in Appendix C, together with some relevant properties of the Fock vacuum associated to that mass matrix.

\section{The Cohomology Argument}

In this section we provide some criteria for the existence of the normalizable supersymmetric ground states of a Hamiltonians with flat directions. These criteria are based on the properties of the ground states of the perturbed Hamiltonian $H_{w} \approx \frac{1}{2}\left\{Q_{w}, \bar{Q}_{w}\right\}$. As it was mentioned above, despite the fact that the local cohomologies of the operators $Q$ and $Q_{w}$ are equivalent, the mere existence of normalizable ground states of the Hamiltonian $H_{w}$ does not prove that they exist also in the original Hamiltonian $H_{0}$. However, they do exists if additional properties are fulfilled. We summarize our method in the following

Lemma 1 There exists a normalizable ground state of the Hamiltonian $H_{0}$ if:

i) The Witten index of the Hamiltonian $H_{w}$ is not zero.

ii) $\psi_{w}^{ \pm} \equiv e^{ \pm w} \phi_{w}$ are normalizable, where $\phi_{w}$ is a normalizable ground state of the perturbed Hamiltonian $H_{w}$.

The condition (i) ensures the existence of a normalizable solution of the perturbed Hamiltonian $H_{w}$.

The functions $\psi_{w}^{ \pm}$are cohomology representatives of the operators $Q, \bar{Q}$, since they obey:

$$
Q \psi_{w}^{+}=\bar{Q} \psi_{w}^{-}=0
$$

On cohomological grounds we may write:

$$
\begin{aligned}
\psi_{w}^{+} & =\alpha+\gamma, \quad \psi_{w}^{-}=\bar{\alpha}+\bar{\gamma} \\
\gamma & \in \overline{\mathcal{I} Q}, \quad \alpha \notin \overline{\mathcal{I} Q} \\
\bar{\gamma} & \in \overline{\mathcal{I} \bar{Q}}, \quad \bar{\alpha} \notin \overline{\mathcal{I} Q} .
\end{aligned}
$$

Here $\overline{\mathcal{I} Q}$ is the closure in $L_{2}$ of the image of $Q$ etc. If both $\psi_{w}^{+}$and $\psi_{w}^{-}$are $L_{2}$, then so are $\alpha$ and $\bar{\alpha}$. By the definition of $\gamma$ and $\bar{\gamma}$, there exist two sequences of $L_{2}$ states, $\beta_{n}, \bar{\beta}_{n}$, such that

$$
\gamma=\lim _{n \rightarrow \infty} Q \beta_{n}, \quad \bar{\gamma}=\lim _{n \rightarrow \infty} \bar{Q} \bar{\beta}_{n}
$$

By computing the scalar product $\left(\psi_{w}^{+}, \psi_{w}^{-}\right)$we find

$$
1=\left(\psi_{w}^{+}, \psi_{w}^{-}\right)=\lim _{n \rightarrow \infty}\left(\alpha+Q \beta_{n}, \psi_{w}^{-}\right)=(\alpha, \bar{\alpha})+\lim _{n \rightarrow \infty}\left(\beta_{n}, \bar{Q} \psi_{w}^{-}\right)=(\alpha, \bar{\alpha})
$$


This equation, which is well defined when $\alpha, \beta_{n}$ etc. are in $L_{2}$, implies that $\alpha$ and $\bar{\alpha}$ cannot be zero. Notice that this result follows only if both $\psi_{w}^{ \pm}$are $L_{2}$.

The non-triviality of the cohomologies ensures the existence of a normalizable ground state of $H_{0}$. This is a well known fact in mathematics; for completeness, we give here below a short proof of this result.

Define two orthogonal projectors, $P_{n}, R_{n}$, such that $P_{n}+R_{n}=1 . P_{n}$ is the spectral projector associated to $H_{0}$, projecting over eigenstates with energy $E \geq 1 / n$, while $R_{n}$ projects over states with energy less than $1 / n$. For all $n>0, P_{n} \alpha$ can be written as $Q \beta_{n}$. By defining $\beta_{n}=\bar{Q} H^{-1} P_{n} \alpha$ (see also ref. [5]); $\beta_{n}$ is $L_{2}$ since the operator $\bar{Q} H^{-1} P_{n}$ is bounded. Analogously, $\bar{\beta}_{n} \equiv P_{n} \bar{\alpha}$ is $\bar{Q}$ exact. Moreover, Since $\alpha$ is $L_{2}$, and $\mathcal{I} R_{n} \subset \mathcal{I} R_{m}$ for $n>m$, the sequence $\alpha_{n} \equiv R_{n} \alpha$ is Cauchy; therefore, it converges to a state $\tilde{\alpha}=\lim _{n \rightarrow \infty} \alpha_{n}$ which, by construction, is normalizable and obeys $H_{0} \tilde{\alpha}=0$. The state $\tilde{\alpha}$ cannot be zero because of eq. (5) and the definition of $\alpha$ given in eq. (3).

Q.E.D.

As we just noticed, if only one of the $\psi_{w}^{ \pm}$is normalizable, it may happen that $\left(Q \beta_{n}, \psi_{w}^{-}\right) \neq$ $\left(\beta_{n}, \bar{Q} \psi_{w}^{-}\right)=0$, so that the cohomology may still be empty. To illustrate this phenomenon, let us consider a simple example: a free supersymmetric particle in one dimension, which is known to have no normalizable ground states. Take a perturbing superpotential $w=\frac{1}{2} x^{2}$. The perturbed problem has a zero energy normalizable solution $\phi_{w}=\exp \left(-\frac{1}{2} x^{2}\right)$, so that $\psi_{w}^{-}$is normalizable, while $\psi_{w}^{+}$is not. What actually happens in this example, is that $\psi_{w}^{-}$is cohomologically trivial, i.e. there exists $\chi$ s.t. $\psi_{w}^{-}=Q \chi$.

Note that the argument in the lemma cannot be reversed: an infinite norm of the $e^{ \pm w} \phi_{w}$ does not imply non-normalizability for $\phi_{0}$.

The Hamiltonian $H_{w}$ should be chosen in such a way that avoids the typical problems associated with flat directions. Particularly simple for the analysis is the case when the potential $V_{w}$ of the supersymmetric Hamiltonian $H_{w}$ has only one isolated minimum. Then, since the Witten index is nonzero, this system is guaranteed to have (at least) a normalizable ground states of zero energy, i.e. there exists (at least) a state $\phi_{w}$ s.t. $H_{w} \phi_{w}=0$, and $\left\|\phi_{w}\right\|<\infty$.

Given that the perturbing potential is a non-singular function, and that the function $\phi_{w}$ is square integrable, the normalizability of the $\psi_{w}^{ \pm}$has to be checked only at infinity, since the integral over a ball of radius $R_{0}$ yields:

$$
\left\|\psi_{w}^{ \pm}\right\|_{\downarrow R_{0}}^{2} \equiv \int_{|\mathbf{R}|<R_{0}} d \mathbf{R}\left|\psi_{w}^{ \pm}\right|^{2} \leq \exp \left(2 \sup _{|\mathbf{R}|<\mathbf{R}_{\mathbf{0}}} w\right)\left\|\phi_{w}\right\|_{\downarrow R_{0}}^{2}<\infty
$$




\section{The H-Monopole QM}

The quantum mechanical system that describes the H-monopoles (better, the "missing states" in the problem [1]) can be thought of as the dimensional reduction of an $\mathrm{N}=2$ field theory in four dimensions, whose degrees of freedom are an Abelian vector multiplet $V$, a neutral scalar multiplet $X$, and two charged chiral multiplets, $\Phi^{ \pm}$, of charge \pm 1 with respect to the $U(1)$ gauge group. The kinetic terms are canonical, while the superpotential is:

$$
W=\Phi^{+} \Phi^{-} X
$$

The scalar potential $V$ is given by the quantum-mechanical reduction of the standard $\mathrm{N}=1$ formula, namely:

$$
V=W_{i} W_{i}^{*}+\frac{1}{4} D^{2}+\left(\left|\phi^{+}\right|^{2}+\left|\phi^{-}\right|^{2}\right) A_{\mu}^{2}
$$

Here, $\phi^{ \pm}$etc. denote the scalars of the chiral multiplets; $W_{i}$ denotes the derivative of the superpotential with respect to the $i$-th scalar, and $A_{\mu}, \mu=1,2,3$ is the component of the vector in the multiplet $V$ along the direction $\mu$. The D-terms are given by

$$
D=\left|\phi^{+}\right|^{2}-\left|\phi^{-}\right|^{2}
$$

Following our general strategy, we add to this superpotential a perturbation $w$, and we proceed to show that a) with the new superpotential $W+w$, there exists a unique point where the (non-negative) scalar potential vanishes, with non-vanishing Hessian, and thus its index $n_{B}-n_{F}=1 ; \mathrm{b}$ ) the perturbation lifts the moduli (valleys) $d W=0$ and does not introduce new valleys; c) given a normalizable ground state of the perturbed problem, $\phi_{w}$, the states $\psi^{ \pm}=$

$e^{ \pm \operatorname{Re} w} \phi_{w}$ are normalizable representatives of the cohomology of the original supersymmetric quantum mechanics.

The perturbation we choose is

$$
w=k X
$$

with $k$ an arbitrary nonzero constant. This perturbation, being linear in the coordinate $X$, does not change the fermionic part of the Hamiltonian.

To prove point a), we have to solve the F-term equations $(W+w)_{i}=0$, and set to zero the D-terms. These equations read:

$$
\phi^{ \pm} x=0, \quad \phi^{+} \phi^{-}+k=0, \quad\left|\phi^{+}\right|^{2}-\left|\phi^{-}\right|^{2}=0 .
$$

Here $x$ is the complex scalar in the supermultiplet $X$. The D-term equation implies $\left|\phi^{+}\right|=\left|\phi^{-}\right|$, while the F-term equations give (modulo a gauge transformation, obviously)

$$
x=0, \quad\left|\phi^{ \pm}\right|=|k|^{1 / 2} ; \quad A_{\mu}=0, \mu=1,2,3 .
$$


We do not need to compute the Hessian to see that it it nonzero. Indeed, since $\phi^{ \pm} \neq 0$, the 4- $\mathrm{d} \mathrm{N}=1$ model is in the Higgs phase: all fields are massive. Upon dimensional reduction, this means that the Hessian of the scalar potential $V$ is nonzero.

To prove point b) it is convenient to rewrite the scalar potential as

$$
V=\frac{1}{4}\left(\left|\phi^{+}\right|^{2}+\left|\phi^{-}\right|^{2}\right)^{2}+|k|^{2}+2 \operatorname{Re} k \phi^{+} \phi^{-}+\left(|x|^{2}+A_{\mu}^{2}\right)\left(\left|\phi^{+}\right|^{2}+\left|\phi^{-}\right|^{2}\right) .
$$

We want to prove that for $R^{2} \equiv\left(\left|\phi^{+}\right|^{2}+\left|\phi^{-}\right|^{2}+|x|^{2}+A_{\mu}^{2}\right) \rightarrow \infty$, the potential attains its minimum value, $|k|^{2}$, along the valleys at $\phi^{ \pm}=0$. The minimization in $\phi^{ \pm}$, keeping $\left(\left|\phi^{+}\right|^{2}+\left|\phi^{-}\right|^{2}\right)$ fixed, gives $\left|\phi^{+}\right|=\left|\phi^{-}\right|$, and allows us to rewrite the potential as

$$
\begin{aligned}
V= & \frac{1}{4} R^{4} \cos ^{4} \theta-|k| R^{2} \cos ^{2} \theta+R^{4} \cos ^{2} \theta \sin ^{2} \theta+|k|^{2} \\
& R^{2} \cos ^{2} \theta \equiv\left|\phi^{+}\right|^{2}+\left|\phi^{-}\right|^{2}, \quad R^{2} \sin ^{2} \theta \equiv|x|^{2}+A_{\mu}^{2} .
\end{aligned}
$$

For $R \rightarrow \infty$, the minimization in $\theta$ gives a minimum at $\cos \theta=0$, i.e. along the flat directions of the unperturbed model.

Point c) is crucial in showing that the perturbed problem has the same cohomology as the unperturbed one. To prove it, we need only study the asymptotic form of the ground state in the perturbed problem, since $w$ is regular (and bounded) on any compact set. To apply Lemma 1, one has to uncover the asymptotic form of the zero energy solution in the perturbed problem at large distances. While unable to solve the problem exactly, we may use the BornOppenheimer approximation in the asymptotic region. Indeed, as it follows from the form of the scalar potential, the frequency of the oscillations in the directions transverse to the moduli subspace $\phi^{ \pm}=0$ is $\omega_{\text {trans. }} \geq \frac{1}{2} R^{2}$, while the characteristic frequency of the oscillations on the moduli is $\omega_{\text {moduli }} \leq|k|$. For $R$ large enough, the separation into "slow" and "fast" modes holds. We can label the 5 -d moduli space with the vector

$$
\mathbf{x} \equiv\left(\operatorname{Re} x, \operatorname{Im} x, A_{1}, A_{2}, A_{3}\right)
$$

and define:

$$
\mathbf{k} \equiv(\operatorname{Re} k, \operatorname{Im} k, 0,0,0)
$$

Along the flat directions, the wave function, $\Psi(\mathbf{x})$, satisfies:

$$
\left(-\frac{1}{2} \nabla_{\mathbf{x}} \nabla_{\mathbf{x}}+\frac{1}{2}|\mathbf{k}|^{2}\right) \Psi(\mathbf{x})=0
$$

Re-writing this equation in spherical coordinates, and denoting by $\omega$ the angular variables, and by $r$ the radius $|\mathbf{x}|$, one obtains:

$$
\left(\frac{d-1}{r} \frac{\partial}{\partial r}+\frac{\partial^{2}}{\partial r^{2}}+\frac{1}{r^{2}} \frac{\partial^{2}}{\partial \omega^{2}}-k^{2}\right) \Psi(\mathbf{x})=0 .
$$


After separation of variables; eq. (18) gives, for the radial part of the wave function, $\Psi(\mathbf{x})=$ $R(r) \Omega(\omega)$ :

$$
\left(\frac{d-1}{r} \frac{\partial}{\partial r}+\frac{\partial^{2}}{\partial r^{2}}-\left(\frac{\mathbf{L}^{2}}{r^{2}}+k^{2}\right)\right) R(r)=0,
$$

where $\mathbf{L}^{2}$ is the square of the total angular momentum. The asymptotic behavior of the solutions to eq. (19) at large $r$ is:

$$
R(r) \stackrel{r \rightarrow \infty}{\longrightarrow} \operatorname{const} \frac{e^{-k r}}{r^{\frac{d-1}{2}}}\left(1+\mathcal{O}\left(\frac{1}{r^{2}}\right)\right)
$$

The wave function in the transverse directions decays at least as fast as that of a harmonic oscillator, and the perturbation $w$, being linear in coordinates, cannot spoil the normalizability.

The normalizability of the "slow" modes functions $\psi^{ \pm}(\mathbf{x})=e^{ \pm R e w} \Psi(\mathbf{x})$ can be checked as follows:

$$
\begin{aligned}
& \int_{|\mathbf{x}|>r_{0}} d \mathbf{x}\left|\psi^{ \pm}(\mathbf{x})\right|^{2}=\mathrm{const} \int_{r>r_{0}} d \mathbf{x} \frac{e^{-2|k| r \pm 2 \mathbf{k x}}}{r^{d-1}} \\
= & \operatorname{const} \Omega_{d-2} \int_{r=r_{0}}^{\infty} d r e^{-2|k| r} \int_{0}^{\pi} d \theta \sin ^{d-2} \theta e^{ \pm 2|k| r \cos \theta}=\mathrm{const} \int_{r=r_{0}}^{\infty} \frac{d r}{r^{\frac{d-1}{2}}} .
\end{aligned}
$$

The integral in (21) converges if $d>3$. Since in the case of the H-monopole the dimension of the moduli space is $d=5$, the criteria for the existence of the normalizable supersymmetric ground state are satisfied.

Our argument proves that there always exists at least a supersymmetric ground state in the H-monopole problem. Barring accidental degenerations, this ground state should also be unique, in agreement with the predictions of S-duality.

\section{The D0 Brane QM}

The conjectured duality between the type IIA string theory and M theory [14] as well as the $\mathrm{M}$ (atrix) model conjecture [2] require that a system of $N$ D0 branes in Type IIA string theory has a unique bound state at threshold for any $N$. In this section, we show that such a state exists for any prime $N$. For $N=2$, the existence of a zero-energy bound state has been proven in ref. [7].

It is convenient to rewrite the D0 supersymmetric QM in a formalism that makes manifest just four of its 16 supersymmetries, as we did for the H-monopole. This is achieved by first reducing the 10-d $S U(N)$ super Yang-Mills theory [15 -which describes $N$ D0 branes- to four dimensions, and then perform another dimensional reduction to the 1-d QM [16]. In the 4-d, $\mathrm{N}=1$ language, the theory is made of a vector superfield, $V$, and 3 chiral superfields, that we shall denote with $\Phi_{i}, i=1,2,3$. 
The bosonic degrees of freedom of the vector multiplet are a 4-d vector $A_{\mu}$ in the adjoint of the gauge group, and some auxiliary fields.

The bosonic degrees of freedom of the scalar multiplets are some auxiliary fields and the complex scalars $\phi_{i}$, also in the adjoint of the gauge group.

The model has canonical kinetic terms, and it is completely determined by its superpotential, which is a holomorphic, gauge-invariant function:

$$
W=\frac{1}{6} \epsilon_{i j k} \operatorname{tr} \Phi_{i}\left[\Phi_{j}, \Phi_{k}\right]
$$

Here $\operatorname{tr}$ denotes a trace over the gauge indices. This choice of superpotential ensures that the model is invariant under 16 supersymmetries, and not just the four which are manifest in this formalism.

The scalar potential of the quantum mechanical model reads, in the $A_{0}=0$ gauge:

$$
V=\left|\frac{\partial W}{\partial \phi_{i}}\right|^{2}+\operatorname{tr}\left[\bar{\phi}_{\bar{\imath}}, \phi_{i}\right]\left[\bar{\phi}_{\bar{\jmath}}, \phi_{j}\right]+\operatorname{tr}\left[A_{\mu}, \phi_{i}\right]\left[A_{\mu}, \bar{\phi}_{\bar{\imath}}\right]+\frac{1}{4} \operatorname{tr}\left[A_{\mu}, A_{\nu}\right]^{2} .
$$

Here, $\mu, \nu=1,2,3$.

\subsection{The Deformation}

To exploit the technique described in Section 2, we must first deform the model by adding to it an appropriate superpotential $w$. Our choice for $w$ is

$$
w=-\frac{1}{2} m \operatorname{tr} \Phi_{i}^{2}
$$

with $m$ a nonzero constant. This perturbation has been introduced in ref. [17; in 4-d it gives a mass $|m|$ to the chiral multiplets.

The zeroes of the perturbed potential are given by the solutions of the following equations, modded out by the action of the gauge group:

$$
\begin{aligned}
{\left[A_{\mu}, A_{\nu}\right] } & =0, \quad\left[A_{\mu}, \phi_{i}\right]=0, \quad \mu=1,2,3, \\
D & =\left[\bar{\phi}_{\bar{\imath}}, \phi_{i}\right]=0, \\
W_{k}+w_{k}=0 & \Rightarrow\left[\phi_{i}, \phi_{j}\right]=m \epsilon_{i j k} \phi_{k} .
\end{aligned}
$$

Eqs. 26,27) set to zero the D- and F-terms. This is equivalent to solve eq. (27) and mod out by the complexified gauge group. The total Witten index is obtained by summing over the contributions to the index of each such solution. These equations have been studied in ref. [17, with the result that their solutions are in one-to-one correspondence with the complex conjugacy classes of $S U(2)$ into the gauge group $G$, that is, with the inequivalent representations of $S U(2)$ 
into the fundamental of $G$. There are three types of representations to be considered, generically: the trivial $\left(\phi_{i}=0\right)$, the irreducibile, and some reducible ones. The trivial representation is given by $\phi_{i}=0$. At this point the chiral multiplets have a 4-d "mass" $|m|$ that, upon dimensional reduction, implies that these modes have a nonzero frequency, and thus do not contribute to the index. This means that at $\phi_{i}=0$ the D0 brane system is effectively three dimensional. If, as suggested in ref. [10], the 3-d D0 brane has no bound states, then the $\phi_{i}=0$ minimum gives a null contribution to the index. In the rest of the paper we will assume that this result holds; later in this section we will report an argument supporting this assumption.

When the gauge group is $S U(N)$, and $N$ is prime, the reducible representations break the gauge group to $U(1)^{N-1}$. The light degrees of freedom parametrize an Abelian theory, which again gives a null contribution to the index.

Finally, the only nonzero contribution to the index comes from the unique irreducible representation of dimension $N$. It breaks $S U(N)$ completely, implying that all degrees of freedom are massive, i.e. that the minimum is at $A_{\mu}=0$ and that the Hessian determinant of the potential is nonzero. To sum up, the irreducible representation gives an isolated minimum contributing 1 to the index, while all other minima give no contribution.

\subsection{The $L_{2}$ Cohomology}

The argument above implies that $n_{B}-n_{F}=1$, i.e. that in the perturbed problem with superpotential $W+w$ there exists at least one normalizable ground state.

Next, following our general strategy outlined in Section 2, we must show that it is possible to associate to the perturbed ground state, $\phi_{w}$, two normalizable representatives, $\psi_{w}^{ \pm}$, of the unperturbed cohomologies $Q$ and $\bar{Q}$.

As in the H-monopole case, we set

$$
\psi^{ \pm}=(\exp \pm \operatorname{Re} w) \phi_{w}
$$

To study the normalizability of $\psi_{w}^{ \pm}$we can limit ourselves to the asymptotic, large $\phi_{i}$ region, since for finite $\phi_{i}$ both $\phi_{w}$ and $\psi_{w}^{ \pm}$are smooth and obviously normalizable.

We want, first of all, to find the new "valleys" of the perturbed potential, $V_{w}$, for large $\phi_{i}$, i.e. to find its minima for fixed, large radius $R=\left(\operatorname{tr} \phi_{i} \bar{\phi}_{\bar{\imath}}\right)^{1 / 2}$. This can be done by adding to the potential a Lagrange multiplier, and looking for the stationary points of

$$
\begin{aligned}
V_{w}+\lambda\left(\operatorname{tr} \phi_{i} \bar{\phi}_{\bar{\imath}}-R^{2}\right)= & \left|\frac{\partial(W+w)}{\partial \phi_{i}}\right|^{2}+\operatorname{tr}\left[\bar{\phi}_{\bar{\imath}}, \phi_{i}\right]\left[\bar{\phi}_{\bar{\jmath}}, \phi_{j}\right]+\operatorname{tr}\left[A_{\mu}, \phi_{i}\right]\left[A_{\mu}, \bar{\phi}_{\bar{\imath}}\right]+\frac{1}{4} \operatorname{tr}\left[A_{\mu}, A_{\nu}\right]^{2}+ \\
& +\lambda\left(\operatorname{tr} \phi_{i} \bar{\phi}_{\bar{\imath}}-R^{2}\right),
\end{aligned}
$$

\footnotetext{
${ }^{7}$ Obviously, the definition of bosons and fermions in supersymmetric QM is conventional, and it can be changed with a unitary redefinition of the fermionic Fock vacuum.
} 
in the limit $R \rightarrow \infty$. Minimization of eq. (29) for large $R$ shows that the valleys of the perturbed potential are the same of the unperturbed one:

$$
\left[\phi_{i}, \phi_{j}\right]=\left[\phi_{i}, \bar{\phi}_{\bar{\imath}}\right]=0
$$

and that along the valleys $V_{w}=|m|^{2} R^{2}$.

Our aim is to prove that the following integral converges:

$$
\int_{\operatorname{tr} \phi_{i} \bar{\phi}_{\bar{\imath}} \geq R^{2}} d \mu \psi_{w}^{ \pm *} \psi_{w}^{ \pm}
$$

Here $d \mu$ denotes the (flat) integration measure of all bosonic variables. For $R \gg 1$, we can use again the adiabatic approximation, and reduce the integral to the estimate of the behavior of the adiabatic wave function on the $9(N-1)$ bmoduli space given by eq. (30).

The asymptotic behavior of the wave function $\phi_{w}$ may be estimated as follows.

On the moduli space, the Born-Oppenheimer wave function must satisfy the equation

$$
H \phi_{w}=0, \quad H=H_{1}+H_{2}, \quad H_{1}=-\triangle_{x}, \quad H_{2}=-\nabla_{y} \nabla_{\bar{y}}+|m|^{2}|y|^{2}+|m|\left[\psi_{\alpha}^{\dagger} \psi_{\alpha}-3(N-1)\right] .
$$

The effective Hamiltonian $H$ is just the reduction of the perturbed D0 brane Hamiltonian to the moduli space. Here, $\triangle_{x}$ is the $3(N-1)$-dimensional Laplacian acting on the bosonic moduli of the vector multiplet: $x \equiv\left(A_{1}, A_{2}, A_{3}\right)$. We parametrized the moduli space of the chiral multiplets $\Phi_{i}$ by $3(N-1)$ superfields $Y=y+\theta^{\alpha} \psi_{\alpha}, \alpha=1,2$.

The asymptotic behavior of $\phi_{w}$ is generically the same as that of the Green function $G=H^{-1}$, even though it may be better, in some exceptional cases. By diagonalizing $H$ and denoting by $K=0, . ., 6(N-1)$ the eigenvalue of the fermion number operator $\psi_{\alpha}^{\dagger} \psi_{\alpha}$ we find:

$$
G\left(x, y, K \mid x^{\prime}, y^{\prime}, K^{\prime}\right)=\delta_{K K^{\prime}} \sum_{n=0}^{\infty} \int \frac{d^{3(N-1)} p}{(2 \pi)^{3(N-1)}} \frac{1}{p^{2}+|m|(n+K)} e^{i p\left(x-x^{\prime}\right)} \Phi_{n}(y)^{*} \Phi_{n}\left(y^{\prime}\right),
$$

where the $\Phi_{n}(y)$ are the usual eigenstates of the $6(N-1)$-dimensional harmonic oscillator with frequency $|m|$. By using the Schwinger representation for the free propagator and integrating in $p$ we find:

$G\left(x, y, K \mid x^{\prime}, y^{\prime}, K^{\prime}\right)=\frac{1}{2}(2 \pi)^{-3(N-1) / 2} \int_{t=0}^{\infty} d t t^{-3(N-1) / 2} \exp \left(-\left|x-x^{\prime}\right|^{2} / 2 t\right)\left\langle y, K\left|\exp \left(-t H_{2}\right)\right| y^{\prime}, K^{\prime}\right\rangle$.

Eq. (34) can be computed with a standard Euclidean functional integral, which gives

$$
\begin{aligned}
G\left(x, y, K \mid x^{\prime}, y^{\prime}, K^{\prime}\right)= & \delta_{K K^{\prime}} \frac{1}{2}(2 \pi)^{-3(N-1) / 2} \int_{0}^{\infty} d t t^{-3(N-1) / 2} \exp \left\{-\left|x-x^{\prime}\right|^{2} / 2 t+|m| t[3(N+\right. \\
& -1)-K]\}\left(\frac{|m|}{\sinh |m| t}\right)^{3(N-1)} \exp \left\{-\frac{|m|}{\sinh |m| t}\left[\left(|y|^{2}+\right.\right.\right. \\
& \left.\left.\left.+\left|y^{\prime}\right|^{2}\right) \cosh |m| t-2 \operatorname{Re}\left(y^{*} y^{\prime}\right)\right]\right\}
\end{aligned}
$$

\footnotetext{
${ }^{8}$ Here we write all formulae for the gauge group $S U(N)$, of course.
} 
The ground-state wave function $\phi_{w}$, and thus $\psi_{w}^{ \pm}$, is not an eigenstate of the fermion number operator $\psi_{\alpha}^{\dagger} \psi_{\alpha}$, as explained in more detail in Appendix B. Rather, $\psi_{w}^{ \pm}$is a superposition of wave functions with different fermion numbers. Asymptotically in $|y|$ one has:

$$
\psi_{w}^{ \pm} \approx \sum_{K=0}^{6(N-1)} c_{K} G\left(x, y, K \mid x^{\prime}, y^{\prime}, K\right) \exp \left( \pm \operatorname{Re} m y^{2}\right),
$$

for some constant coefficients $c_{K}$. The asymptotic decay rate of the Green function (35) increases with $K$, which implies that, for large $|y|$ :

$$
\psi_{w}^{ \pm} \approx c_{K^{o}} G\left(x, y, K^{o} \mid x^{\prime}, y^{\prime}, K^{o}\right) \exp \left( \pm \operatorname{Re} m y^{2}\right)
$$

where $K^{o}$ is the smallest $K$ appearing in equation (36) with a nonzero coefficient $c_{K}$. To prove that the $\psi_{w}^{ \pm}$are normalizable we need only study the large $\phi_{i}$ region, i.e. $|y| \gg\left|y^{\prime}\right|$. We first integrate in $x$ and obtain

$$
\begin{aligned}
\int d^{3(N-1)} x\left|\psi_{w}^{ \pm}(x, y)\right|^{2} \approx & \frac{\left|c_{K^{o}}\right|^{2}}{2} \int_{0}^{\infty} d s \int_{0}^{\infty} d t(t+s)^{-3(N-1) / 2} \exp \{|m|(t+s)[3(N-1)+ \\
& \left.\left.-K^{o}\right]\right\}\left(\frac{|m|^{2}}{\sinh |m| s \sinh |m| t}\right)^{3(N-1)} \exp [-|m|(\operatorname{coth}|m| s+ \\
& \left.+\operatorname{coth}|m| t)|y|^{2} \pm 2 \operatorname{Re} m y^{2}\right] .
\end{aligned}
$$

We can restrict the region of integration to large values of $y$ by restricting $y$ to lie outside of the hypercube $\mathcal{C}$, defined by $\operatorname{Re} y_{i}, \operatorname{Im} y_{i} \leq R \forall i$, with $R \gg|m|^{1 / 2}$. We find:

$$
\begin{aligned}
\int_{R^{6(N-1)}-\mathcal{C}} d^{6(N-1)} y\left|\psi_{w}^{ \pm}\right|^{2} \approx & A \int_{0}^{\infty} d s \int_{0}^{\infty} d t(t+s)^{-3(N-1) / 2} \exp \left\{|m|(t+s)\left[3(N-1)-K^{o}\right]\right\} \times \\
& \left\{\sinh ^{2}|m| s \sinh ^{2}|m| t\left[(\operatorname{coth}|m| s+\operatorname{coth}|m| t)^{2}-4\right]\right\}^{-3(N-1) / 2} \times \\
& \exp \left[-R^{2}|m|(\operatorname{coth}|m| s+\operatorname{coth}|m| t-2)\right],
\end{aligned}
$$

where $A$ is a finite, positive constant. The dangerous region of integration for the norm of $\psi_{w}^{ \pm}$ is where $s$ and $t$ are both large. There, eq. (39) simplifies considerably, and one finds

$$
\begin{aligned}
\int_{R^{6(N-1)}-\mathcal{C}} d^{6(N-1)} y\left|\psi_{w}^{ \pm}\right|^{2} \approx & A \int_{M}^{\infty} d s \int_{M}^{\infty} d t(t+s)^{-3(N-1) / 2} \exp \left\{|m|\left[3(N-1) / 2-K^{o}\right](s+t)\right\} \\
& \times[\cosh |m|(t-s)]^{-3(N-1) / 2}+B=\int_{2 M}^{\infty} d u \int_{-\infty}^{\infty} d v u^{-3(N-1) / 2} \times \\
& \exp \left\{|m|\left[3(N-1) / 2-K^{o}\right] u\right\}(\cosh |m| v)^{-3(N-1) / 2}+B .
\end{aligned}
$$

Here $M \gg 1 /|m|, u=s+t, v=s-t$, and $B$ is another finite, positive constant. As explained in Appendix $\mathrm{C}$, the ground-state wave function is the completely filled state in the Fock space generated by a subset of the oscillators $\psi_{\alpha}, \psi_{\alpha}^{\dagger}$. Using this result, it is easy to compute the 
fermion number $K^{o}$. Indeed, for each $2 \leq l \leq 2 j$ there exist $2 \times 3$ such oscillators, while for $l=1$ there are only two such oscillators ${ }^{9}$; therefore,

$$
K^{o}=\sum_{l=2}^{2 j} 6+2=6(2 j-1)+2=6 N-10 .
$$

The integral in eq. (39) is finite if and only if $K^{o}>3(N-1) / 2$. This happens for $N>17 / 9$, and therefore the cohomology representatives $\psi_{w}^{ \pm}$are $L_{2}$ for all $S U(N)$ with $N$ prime.

By applying the method of Section 2, we conclude that a system of $N$ D0 brane in 9 dimensions has at least a zero-energy bound state for any prime $N$.

This conclusion, in agreement with expectations from string duality and Matrix theory, holds if no bound states exist in the 3-d D0 brane system. An argument which shows that this is the case has been formulated by S. Shenker. It goes as follows. The asymptotic moduli space can be partitioned into $N-1$ subregions in which the VEVS of the scalars break $S U(N)$ to $S U(N-n) \times U(1)^{n}, n=1, . ., N-1$. Geometrically, this corresponds to have $N-n$ D0 branes close to each other and $n$ far away. Along this flat direction, the ground-state wave function approximately obeys a free $d n$-dimensional Laplace equation, whose long-distance behavior is generically given by the Green function $G(x, 0)=|x|^{2-d n}, x \in R^{d n}$. This function is square summable at large $x$ only if $n d>4$; for $n=1$ one gets $d>4$. This argument is incomplete (in some cases the asymptotic behavior of the wave function can be better than that of the Green function), but still rather compelling, especially when coupled with the rigorous results of [9].

\section{Conclusions}

In this paper, we proposed a novel way of studying the existence of bound states at threshold in supersymmetric QM systems. We applied our method to the case of H-monopoles, and rederived a known result, namely that there is (at least) one normalizable bound state at threshold in the $U(1)$ theory describing the "missing" H-monopoles $\mathbb{1 1}$.

We also studied a $\mathrm{QM}$ with gauge group $S U(N)$ and 16 supercharges, that describes a system of $N$ D0 branes in nine dimensions. Applying our method we found that this system possesses (at least) a bound state at threshold for any prime $N$, assuming that no bound states of D0 branes exist in three dimensions. This result complements the one of ref. [7], in which the existence of a bound state was proven for $N=2$. We believe that the restriction on $N$ is purely technical and not due to any fundamental limitation of our method.

Our method uses the stability of the supercharge cohomology under appropriate deformations of the superpotential. Compared with the technique of refs. [6, 7], it has the advantage

\footnotetext{
${ }^{9}$ See Appendix $\mathrm{C}$ for notations. The factor 2 comes from the index $\alpha=1,2$; the factor 3 comes from the index $s_{3}=0, \pm 1$. For $l=1$ only $s_{3}=0$ is allowed.
} 
that it does not require the computation of a massive multi-dimensional "bulk" integral, and the delicate estimate of a boundary term.

Besides these technical points, we think that our method may be of interest since it seems possible to extended it to the computation of the index of more complicated (and less supersymmetric) QM models, such as the QM describing 4-d BPS black holes described in [11.

Finally, let us emphasize that our method always determines wave functions which are in the same cohomology class of the true ground state, rather than the ground state itself. This means, in particular, that our method does not determine the true asymptotic behavior of the groundstate wave function, which may be very different from that of the cohomology representatives.

\section{Acknowledgments}

We would like to thank J. Cheeger for useful discussions, and M. Stern for useful comments on the manuscript. M.P. is supported in part by NSF grants no. PHY-9318781, PHY-9722083; A.R. is supported in part by a Margaret and Herman Sokol Research Fellowship. A.R. would like to thank the LPTHE at the University of Paris VI for its kind hospitality and support.

\section{Appendix A}

The deformed Hamiltonian $H_{w}$ may still turn out to be too complicated to be solved exactly. However, we are interested only in the asymptotic behavior of the solutions, which can be found in the Born-Oppeheimer approximation.

Typically, since the perturbation is small compared to the original Hamiltonian away from the subspace of flat directions, and the valleys in the original problem become increasingly narrow, the frequency of the oscillations transverse to the original valleys is very large compared to the frequency along the valleys. Given that, one separates the variables into "slow" $\xi$, and "fast" $\eta$, and introduces the corresponding Hamiltonians:

$$
H(\xi, \eta)=H_{1}(\xi, \eta)+H_{2}(\xi)
$$

The $H_{1}$ piece depends on the slow variables $\xi$ only parametrically, i.e. it contains no derivatives with respect to $\xi$.

The eigenfunctions of the full Hamiltonian satisfy

$$
\left(H_{1}+H_{2}\right) \Phi(\xi, \eta)=E(\xi, \eta) \Phi(\xi, \eta)
$$

and in adiabatic approximation are factorized as:

$$
\Phi(\xi, \eta)=\sum \Xi(\xi) \Psi(\xi, \eta)
$$


The $\Psi(\xi, \eta)$ are the eigenfunctions of $H_{1}$ :

$$
H_{1}(\xi, \eta) \Psi(\xi, \eta)=E^{\prime}(\xi) \Psi(\xi, \eta) .
$$

Both Hamiltonians $H_{1,2}$ are supersymmetric and hence non-negative, and for that reason in order to find the ground state of (A.2), we have to look for zero energy solutions of the corresponding Hamiltonians. The existence of a solution to (A.2) is implied by the non-vanishing of the Witten index, and we are only concerned about the asymptotic form of the solutions. We want to show that the ground state $\Psi^{(0)}(\xi, \eta)$ of eq. A.4), and the ground state $\Xi^{(0)}(\xi)$, solution of

$$
\mathrm{H}_{2} \Xi^{(0)}(\xi)=0
$$

give an adequate asymptotic approximation of the ground state wave function $\Phi^{(0)}(\xi, \eta)$.

Recall that the asymptotic form of a solution of our Fredholm differential operators is not affected by terms of order (distance) ${ }^{-2}$. The zeroth order terms give the leading exponent, while the terms of order (distance $)^{-1}$ provide the subleading power correction. Therefore, when solving eq. (A.2), one may neglect corrections of order (distance) $)^{-2}$ in the equation for $\Phi(\xi, \eta)$.

Since $H_{2}(\xi)=\frac{1}{2}\left\{Q_{\xi}, \bar{Q}_{\xi}\right\}$ is a supersymmetric Hamiltonian, one has $Q_{\xi} \Xi^{(0)}(\xi)=\bar{Q}_{\xi} \Xi^{(0)}(\xi)=$ 0 . Therefore:

$$
\left(H_{1}(\xi, \eta)+H_{2}(\xi)\right) \Xi^{(0)}(\xi) \Psi^{(0)}(\xi, \eta)=-\Xi^{(0)}(\xi)\left(\triangle_{\xi} \Psi^{(0)}(\xi, \eta)\right) .
$$

Multiplying the r.h.s of eq. (A.6) by $\Psi^{(0)^{*}}(\xi, \eta)$, and integrating over $\eta$, one obtains:

$$
\Xi^{(0)}(\xi) N^{2}(\xi) \int d \eta \Psi^{(0)^{*}}(\xi, \eta)\left(\frac{\partial^{2}}{\partial \xi^{2}}+\frac{d_{\xi}-1}{\xi} \frac{\partial}{\partial \xi}\right) \Psi^{(0)}(\xi, \eta)
$$

The variable $\xi$ is treated as a parameter in the equation $H_{1}(\xi, \eta) \Psi^{(0)}(\xi, \eta)=0$. The function $\Psi^{(0)}(\xi, \eta)$ is of the form

$$
\Psi^{(0)}(\xi, \eta)=N(\xi) \mathcal{F}\left(\xi^{\alpha} \eta\right),
$$

where $N$ is a normalization factor, and $\alpha(\eta) \geq \alpha_{0}>0$ depends on the particular potential. For a harmonic oscillator, $\alpha$ is of course $\frac{1}{2}$. After a change of variables to $\mu \equiv \xi^{\alpha} \eta$, eq. (A.7) takes the form:

$$
\Xi^{(0)}(\xi) N^{\prime 2} \int d \mu \mathcal{F}^{*}(\mu)\left(\frac{\partial^{2}}{\partial \xi^{2}}+\frac{d_{\xi}-1}{\xi} \frac{\partial}{\partial \xi}\right) \mathcal{F}(\mu),
$$

the where $N^{\prime}$ is just a number:

$$
\frac{1}{N^{\prime 2}}=\int d \mu|\mathcal{F}(\mu)|^{2}
$$

Due to the definition of $\mu$, each differentiation in eq. (A.9) brings down one power of $\xi$, so that eq. (A.7) becomes:

$$
\frac{\Xi^{(0)}(\xi)}{\xi^{2}} \times(\text { integral independent of } \xi) .
$$

Consequently, $\Phi^{(0)}(\xi, \eta)=\Xi^{(0)}(\xi) \Psi^{(0)}(\xi, \eta)$, obtained from eqs. (A.4, A.5), gives the ground state of eq. (A.1) up to order $\xi^{-2}$. 


\section{Appendix B}

In this appendix, we want to study the semiclassical approximation for a supersymmetric quantum mechanics.

In this approximation, the zero-energy wave function takes the form

$$
\Psi(x)=\exp [-S(x) / \hbar] F(x)+O(\hbar) .
$$

Here $x$ denotes all bosonic variables, while $F(x)$ is a map from $x$ into the fermionic Fock space. The zero-energy ground state of a supersymmetric system obeys a Schrödinger equation which reads, schematically:

$$
\left[-\frac{\hbar^{2}}{2} \nabla_{x}^{2}+V(x)+\hbar M(x)\right] \Psi(x)=0 .
$$

$V(x) \geq 0$ is the scalar potential, which by assumption has an isolated, non-degenerate zero at $x=0$, while the "fermion mass" term $M(x)$ is a map from $x$ into the linear operators of the Fock space, i.e. it is an $x$-dependent finite-dimensional matrix. By substituting the ansatz eq. (B.1) into the Schrödinger equation and expanding in powers of $\hbar$, we find the equations:

$$
\frac{1}{2} \nabla_{x} S \nabla_{x} S-V=0, \quad\left[\left(\nabla_{x} S\right) \nabla_{x}+M(x)+\frac{1}{2}\left(\nabla_{x}^{2} S\right)\right] F(x)=0 .
$$

The first equation is the familiar Hamilton-Jacoby equation of a bosonic system with potential $-V(x)$, while the second determines the vector $F$ in the Fock vacuum. By denoting with $N(x)$ the matrix $M(x)+I\left(\nabla_{x}^{2} S\right) / 2$ ( $I$ is the identity in the Fock space), we can easily write down the solution for $F(x)$ as

$$
F(x)=\lim _{\tau \rightarrow-\infty} T \exp \left[-\int_{\tau}^{t(x)} N(x(s))\right] F(0) .
$$

Here, $T$ denotes the ordering of the matrices $N$ in the parameter $s . x(s)$ is the zero-energy classical trajectory approaching $x=0$ at $s \rightarrow-\infty$, and reaching $x$ at $s=t(x)$. A general property of a supersymmetric system is that the eigenvalues of $N(x)$ are non-negative near $x=0$. In particular, $N(0)$ has a unique zero eigenvalue, $|0\rangle$, which may be used to define the Fock vacuum. Notice also that the trajectory $x(s)$ in eq. (B.4) spends an infinite amount of time near $x=0$. This fact, together with the semipositive-definitness of $N$, implies that all components of $F(0)$ are projected out by the operator $\lim _{\tau \rightarrow-\infty} T \exp \left[-\int_{\tau}^{t(x)} d s N(x(s))\right]$, except the one parallel to the Fock vacuum $|0\rangle$. Therefore, we can choose as initial condition in eq. (B.4) $F(0)=$ const $|0\rangle$. Since $N(x(s))$ has fermion number zero, $F(x)$ has the same fermion number as $F(0)$, i.e. it is proportional to the Fock vacuum defined by $N(0)$. This last remark is important, since by using another $N(x)$ one may define another Fock vacuum, which may not coincide with the previous one. In terms of the new Fock vacuum, the old one (generically) is not even an eigenstate of the new fermion number operator. For the case of the Do brane, this was explicitly shown in ref. [3]. 


\section{Appendix C}

The second derivative of the superpotential of the perturbed D0 brane system is:

$$
(W+w)_{i j}^{\prime \prime}(\phi)=\epsilon_{i l j} \phi_{l}-m \delta_{i j} I
$$

where $I$ is the identity matrix acting (as $\phi_{l}$ does) on the adjoint of $S U(N)$. It determines the frequencies of the fermionic oscillators in the chiral multiplets, since the fermionic Hamiltonian is

$$
H_{F}=\epsilon_{\alpha \beta} \psi_{\alpha}^{i}(W+w)_{i j}^{\prime \prime}(\phi) \psi_{\beta}^{j}+\text { h.c. }+ \text { gaugino terms, } \quad \alpha, \beta=1,2 \text {. }
$$

The superpotential $W+w$, given by eqs. (22,24) has an isolated stationary point determined by eqs. (25-27). Thanks to eq. (27) the $\phi_{i}$ obey, up to a rescaling, the commutation relations

of the generators of $S U(2)$. Moreover, as discussed in the text, they act on the fundamental of $S U(N)$ as the irreducible representation of dimension $N$. This means that one can define

$$
\phi_{i}=-i m L_{i}
$$

$L_{i}$ acts on the adjoint of $S U(N)$, that is on the traceless product of two fundamentals. In terms of representations of $S U(2), L_{i}$ acts on the reducible representation

$$
j \times j-0=2 j+(2 j-1)+\ldots+1, \quad j=(N-1) / 2 .
$$

Noticing that $i \epsilon_{i l j}$ acts on the index $i$ as the $j=1$ representation of $S U(2)$, we can re-write eq. (C.1) in a remarkably simple way:

$$
(W+w)^{\prime \prime}=-m\left(S_{i} L_{i}+1\right) .
$$

In this equation, $S_{i}$ are the generators of $S U(2)$ in the $j=1$ representation.

Eq. (C.5) is easily diagonalized in terms of the eigenstates of the "total angular momentum" $\vec{J}^{2}=(\vec{S}+\vec{L})^{2}$. The eigenvalues and their multiplicity are given in Table 1 .

Table 1: Eigenvalues of $(W+w)^{\prime \prime}$

\begin{tabular}{|c|c|c|c|}
\hline$\vec{L}$ irrep & $\vec{J}$ irrep & Eigenvalue & Multiplicity \\
\hline \hline $1 \leq l \leq 2 j$ & $l+1$ & $-m(l+1)$ & $2 l+3$ \\
\hline$l$ & $l$ & 0 & $2 l+1$ \\
\hline$l$ & $l-1$ & $m l$ & $2 l-1$ \\
\hline
\end{tabular}

The zero eigenvalues of $(W+w)^{\prime \prime}$ are due to gauge invariance, while the corresponding eigenvectors mix with the gauginos to give states with "fermion mass" $\pm m$. 
For our purpose, it is useful to choose $m$ to be real and positive, and use gauge invariance to put $L_{3}$ in eq. (C.4) in the form

$$
L_{3}=\operatorname{diag}(j, j-1, \ldots,-j)
$$

With this choice, the fields in the Cartan subalgebra of $S U(N)$ are diagonal, up to an $S U(N)$ transformation. Also, at a generic point in moduli space, all and only the fields in the Cartan subalgebra are light. This suggests to introduce another basis for the chiral multiplets, labeled by the eigenvalues of $L_{3}$ and $S_{3}, l_{3}, s_{3}$. In this basis the "light" fermions (i.e. the slow modes in the adiabatic expansion) $\operatorname{read} U^{\dagger} \psi_{l_{3}=0, s_{3}, \alpha} U, U \in S U(N)$, while the heavy fermions (fast modes) read $U^{\dagger} \psi_{l_{3} \neq 0, s_{3}, \alpha} U$. Now, we have two basis with which to label the fermions of the chiral multiplet. One, labeled by the eigenvalues of $\vec{J}^{2}, J_{3}$ diagonalizes the fermion mass matrix around the stationary point given by eqs. (25-27). The other, labeled by the eigenvalues of $L_{3}, S_{3}$, diagonalizes the fermion mass matrix along the moduli space. As shown in Appendix B, the fermionic wave function, $F(\phi)$, is proportional to the Fock vacuum determined by $N(0)$. In our case, this means, among other things, that $F(\phi)$ obeys the following equation:

$$
\alpha_{j_{3}}^{(l-1)} F(\phi)=0 \Rightarrow U^{\dagger} \alpha_{j_{3}}^{(l-1)} U F(\phi)=0, \quad-1 \leq j_{3} \leq 1
$$

Here, we have used the gauge invariance of $F(\phi)$, and denoted by $\alpha_{j_{3}}^{(l-1)}$ the fermion annihilation operator in the basis $\vec{J}^{2}, J_{3}$, belonging to the $\vec{J}$ representation $(l-1)$. When expressed in terms of the creators and annihilators $\beta_{l_{3} s_{3}}^{\dagger}, \beta_{l_{3}, s_{3}}$, in the basis $L_{3}, S_{3}$, this operator obeys:

$$
U^{\dagger} \alpha_{j_{3}}^{(l-1)} U=\left\langle l-1, j_{3} \mid l, 1,0, j_{3}\right\rangle \beta_{0, j_{3}}^{\dagger}+\sum_{m \neq 0}\left\langle l-1, j_{3} \mid l, 1, m, j_{3}-m\right\rangle \beta_{m, j_{3}-m}^{\#}
$$

Here $\beta^{\#}$ stands for either $\beta$ or $\beta^{\dagger}$, while $\left\langle l-1, j_{3} \mid l, 1, l_{3}, s_{3}\right\rangle$ are Clebsch-Gordan coefficients.

Notice the presence of the creation operator $\beta_{0, j_{3}}^{\dagger}$ on the r.h.s. of eq. (C.8). This is a crucial point that needs some explaining. The matrix $(W+w)_{i j}$ in eq. (C.1), computed at the stationary point is real, since it is given by eq. (C.4). This implies that if $\psi_{1}$ and $\psi_{2}$ are two eigenvectors of $(W+w)^{\prime \prime}$, with eigenvalue $\omega$, then the eigenstates of the "fermion mass" are $\psi_{ \pm}=\psi_{1} \pm \psi_{2}^{\dagger}$, with eigenvalues $\pm \omega$. Along the valley given by eq. (30), $(W+w)^{\prime \prime}$ can also be diagonalized. Let us label by $\tilde{\psi}_{1}^{a}, \tilde{\psi}_{2}^{a}$ a basis of eigenstates with eigenvalues $\omega^{a}$. Expanding $\psi_{\alpha}$ in this new basis we find $U^{\dagger} \psi_{\alpha} U=\sum_{a} c_{a} \tilde{\psi}_{\alpha}^{a}, U \in S U(N)$. We also have: $U^{\dagger} \psi_{ \pm} U=\sum_{a}\left(c_{a} \tilde{\psi}_{1}^{a} \pm c_{a}^{*} \tilde{\psi}_{2}^{a \dagger}\right)$. When, say, $\omega^{1}$ and $c_{1}$ are real, one may rewrite this equation in the form $U^{\dagger} \psi_{ \pm} U=c_{1} \tilde{\psi}_{ \pm}^{1}+\ldots$. In this last equation, $\psi_{+}$and $\tilde{\psi}_{+}^{1}$ are both creators or both destructors if $\omega$ and $\omega^{1}$ have the same sign. When $\omega$ and $\omega^{1}$ have opposite sign, instead, $\psi_{+}$is a creator when $\tilde{\psi}_{+}^{1}$ is a destructor, and vice versa. In our case, $c_{1}$ is a Clebsch-Gordan coefficient, which is indeed real; the eigenvalue $\omega$ is $m l$, while $\omega^{1}$ is $-m$, which explains eq. (C.8). 
By writing eq. (C.7) in terms of the oscillators $\beta$, we get an equation that can be simplified by noticing that on the moduli space, away from the origin $\phi_{i}=0$, the charged fermions created by $\beta_{m, s_{3}}^{\dagger}, m \neq 0$ have a very large frequency $\propto\left|\phi_{i}\right|$. This implies that all components of $F(\phi)$, except the one proportional to the ground state of these oscillators, decay as fast as $\exp \left(-\right.$ const $\left.\left|\phi_{i}\right|^{4}\right)$. Eq. (C.7) can thus be rewritten as

$$
\left(\left\langle l-1, j_{3} \mid l, 1,0, j_{3}\right\rangle \beta_{0, j_{3}}^{\dagger}+\sum_{m \neq 0} c_{j_{3}}^{m} \beta_{m, j_{3}-m}^{\dagger}\right) F(\phi)=0 .
$$

Here $c_{j_{3}}^{m}=\left\langle l-1, j_{3} \mid l, 1, m, j_{3}-m\right\rangle$, if $\beta_{m, j_{3}-m}^{\#}=\beta_{m, j_{3}-m}^{\dagger}$, and $c_{j_{3}}^{m}=0$, otherwise. Notice that the sum in eq. (C.9) contains at most 2 non-vanishing terms. Since $F(\phi)$ has zero charged-fermion number, in the large- $\phi_{i}$ region of moduli space all terms in the sum have to vanish separately. Since $\left\langle l-1, j_{3} \mid l, 1,0, j_{3}\right\rangle$ vanishes only for $j_{3}= \pm l$, this may only happen when all $c_{j_{3}}^{m}$ vanish, and $\beta_{0, j_{3}}^{\dagger} F(\phi)=0$ for all $\left|j_{3}\right| \leq \min [1, l-1]$. This equation means that $F(\phi)$, is the completely filled state in the Fock space of the oscillators $\beta_{0, j_{3}}^{\#},\left|j_{3}\right| \leq \min [1, l-1]$. 


\section{References}

[1] E. Witten, Nucl. Phys. B460 (1996) 541, hep-th/9511030.

[2] T. Banks, W. Fishler, S.H. Shenker and L. Susskind, Phys. Rev. D55 (1997) 5112, hep-th/9610043.

[3] B. de Wit, J. Hoppe and H. Nicolai, Nucl. Phys. B305 (1988) 545.

[4] B. de Wit, M. Luscher and H. Nicolai, Nucl. Phys. B320 (1989) 135.

[5] E. Witten, Nucl. Phys. B202 (1982) 253.

[6] S. Sethi and M. Stern, Phys. Lett. B398 (1997) 47, hep-th/9607145.

[7] S. Sethi and M. Stern, hep-th/9705046.

[8] P. Yi, hep-th/9704098.

[9] J. Frölich and J. Hoppe, hep-th/9701119.

[10] B. de Wit, hep-th/9701169.

[11] I.R. Klebanov and A.A. Tseytlin, Nucl. Phys. B475 (1996) 179, hep-th/9604166.

[12] J. Hoppe, hep-th/9709132; hep-th/9709217.

[13] S. Ferrara, Lett. Nuovo Cim. 13 (1975) 629.

[14] E. Witten, Nucl. Phys. B443 (1995) 85, hep-th/9503124.

[15] L. Brink, J.H. Schwarz and J. Scherk, Nucl. Phys. B121 (1977) 77.

[16] M. Claudson and M.B. Halpern, Nucl. Phys. B250 (1985) 689.

[17] C. Vafa and E. Witten, Nucl. Phys. B431 (1994) 3, hep-th/9408074. 